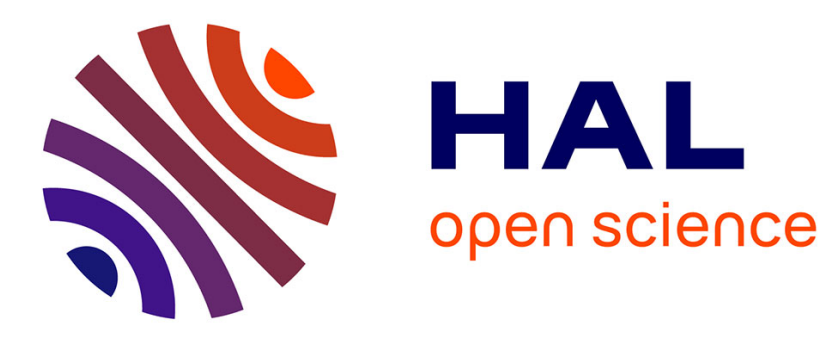

\title{
Developing a Multi-Agent System to Support Multi-Variant Production Ramp-Up Management
}

\author{
Khaled Medini, David Romero, Thorsten Wuest
}

\section{To cite this version:}

Khaled Medini, David Romero, Thorsten Wuest. Developing a Multi-Agent System to Support MultiVariant Production Ramp-Up Management. Smart and Sustainable Manufacturing Systems, 2021, 5 (1), pp.20200082. 10.1520/SSMS20200082 . emse-03233783

\section{HAL Id: emse-03233783 \\ https://hal-emse.ccsd.cnrs.fr/emse-03233783}

Submitted on 8 Nov 2021

HAL is a multi-disciplinary open access archive for the deposit and dissemination of scientific research documents, whether they are published or not. The documents may come from teaching and research institutions in France or abroad, or from public or private research centers.
L'archive ouverte pluridisciplinaire HAL, est destinée au dépôt et à la diffusion de documents scientifiques de niveau recherche, publiés ou non, émanant des établissements d'enseignement et de recherche français ou étrangers, des laboratoires publics ou privés. 


\title{
Developing a Multi-Agent System to Support Multi-Variant Production Ramp-up Management
}

\author{
Khaled Medini ${ }^{\mathrm{a}}$, David Romero ${ }^{\mathrm{b}}$, Thorsten Wuest ${ }^{\mathrm{c}}$ \\ ${ }^{a}$ Mines Saint-Etienne, Univ Clermont Auvergne, INP Clermont Auvergne, CNRS, UMR 6158 LIMOS, F - \\ 42023 Saint-Etienne France \\ ${ }^{b}$ Tecnológico de Monterrey, Mexico City, Mexico \\ ${ }^{b}$ Industrial and Management Systems Engineering, West Virginia University, USA
}

\section{Introduction}

The digital transformation of the manufacturing industry is at full speed, and customers' preferences are changing rapidly whilst their demands increase. This has led to shortening product lifecycles and continuous development and introduction of products into the market. Such a situation forces enterprises to rethink their manufacturing systems to address the resulting challenges of increased product variety and complexity stemming from mass-customized products, as well as market dynamics. To remain competitive, companies need to become more agile and flexible, while steadily increasing the efficiency of their manufacturing operations. In fact, agile and service-oriented companies are disrupting the marketplace, and customers are starting to get used to reliable next-day deliveries (i.e., 'the Amazon effect') in addition to mass-customized or even personalized product offerings. ${ }^{1}$

To keep up with the evolving technological, social and market paces, companies are confronted with the need for frequent ramp-ups to move from product development to stable production. ${ }^{2}$ Ramp-up activities are, however, challenged by increasing product and process complexities resulting from an increasingly diverse offering. When manufacturing companies launch a new product into the market, they not only need to think about the time-to-market as their key measure of success but also take into account the time-to-volume, time-to-quality and time-to-cost required to satisfy their customers' expectations. , $^{3,4,5,6,7}$

Conversely, ramp-up management has not yet received the deserved attention in comparison to other product lifecycle management stages, in particular when it comes to multi-variant 
production. ${ }^{6,7,8}$ In fact, product development and stable or volume production are addressed widely and separately. One major recurring question a decision-maker is likely to consider during product development is how to move from a prototype up to a production ceiling. Such a question involves examining different potential criteria for measuring ramp-up success, such as time, cost and quality. ${ }^{6,7}$ It becomes apparent that there are several general strategies and high-level frameworks available in recent ramp-up management literature. ${ }^{6,7,9}$ However, there is a scarcity of data-backed tools and frameworks that deal with the question of how to support the efficient ramp-up of multivariant production.

This paper aims to bridge this gap by introducing a Multi-Agent System (MAS) supporting rampup management strategies assessment in multi-variant production contexts. The aim is to support decision-makers in the planning stage of the production ramp-up. The MAS relies on the concept of an agent which is an autonomous entity operating in a society of agents in order to contribute to a general goal.

The remainder of the paper is organized as follows: An overview of the research motivation and problem definition is provided in Section 2 and based on this, a review of MAS applications for ramp-up management is given in Section 3. The development of MAS is detailed in Section 4, followed by a case study for evaluating the applicability of the MAS which is presented and discussed in Section 5. Further discussion and perspectives are summarized in Section 6 and the paper ends with concluding remarks in Section 7.

\section{Motivations and Problem Definition}

Individualized customer demands and market expansion is pushing companies to seek not only cost efficiency but also differentiation as a competitive advantage.,10,11 This trend introduces certain obstacles to the successful development and introduction of highly differentiated products into the market. In fact, differentiation comes with associated complexity and costs which may negatively impact time-to-market and time-to-volume. The ramp-up phase is generally set up when moving from the product development to the production phase of the product lifecycle (see Figure $1)$. 


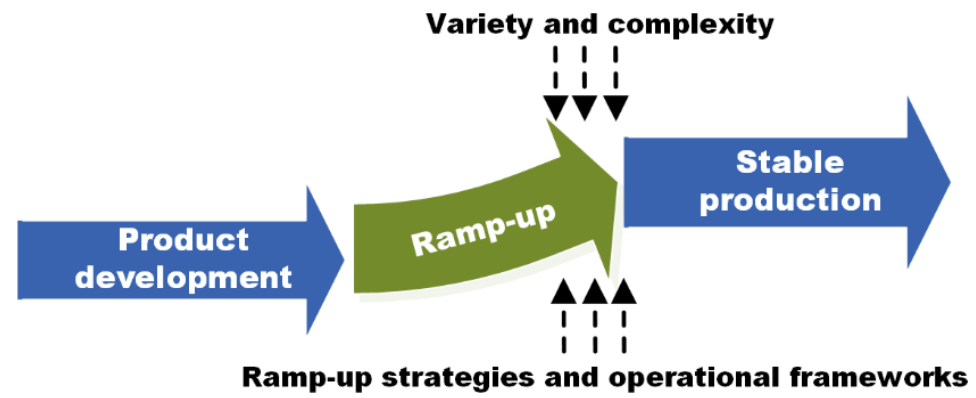

Figure 1. Ramp-up Management in Product Lifecycle11

According to Schuh et al. $(2008)^{12}$, production ramp-up is a value creation phase starting with the completion of product and process design and ending with the achievement of the full production capacity of the manufacturing system, in other words, a successful production run. Ramp-up management is also required when companies experience a significant peak in an existing product or service demand. ${ }^{13}$ A good example is the global demand for Personal Protective Equipment (PPE) that increased tremendously and rapidly during the COVID-19 pandemic outbreak. ${ }^{14,15}$ The current mode of production that includes outsourcing and global sourcing has shown significant limitations at a time when resilience and agility became key denominators of a delocalized industry during this global crisis (e.g., changing priorities, very tough competition) ${ }^{16}{ }^{16}$ Therefore, proper decision-making support combined with innovative approaches is needed for quick production ramp-up and even ramp-down under these circumstances.

Ramp-up and capacity management have therefore started to receive a great deal of attention and have become more critical than ever. More generally, the increasing versatility in today's manufacturing environment started to bring back the production ramp-up concept to the cuttingedge production research trends. This is exemplified by several recent research works. $7^{6,7,17,18,19,20}$ However, the available scientific literature dealing with production ramp-up, particularly in highvariety or even multi-variant production, focuses on relevance and general strategies of production ramp-up management. ${ }^{6}$ There is a lack of operational methods and tools intended for the effective guidance of the decision-making process within manufacturing companies. ${ }^{6,7}$

Glock et al. (2012) ${ }^{20}$ develop a mathematical model to support production planning during rampup considering the learning effect on the production rate. The model allows for optimizing procurement and inventory costs while attempting to match demand. Hansen and Grunow (2015) ${ }^{21}$ propose a similar model which also considers capacity expansion through experience gained in 
production. Therefore, effective capacity during the ramp-up phase is volume-dependent in their model. However, multi-variant production is not addressed by the proposed models, which generally focus on a single product context.

Schuh et al. $(2015)^{17}$ propose a structured approach to support the ramp-up of assembly systems when faced with complex challenges. Their approach relies on a qualitative assessment of complexity drivers and enablers to ensure a cost-efficient and timely migration to serial production. The approach provides significant decision-making support to help manage ramp-up projects and for production management. Fjällström et al. (2007) ${ }^{22}$ highlight the importance of information for a ramp-up, in particular, for handling crises that may occur after the start of production (i.e., disturbances, quality problems, lack of operators, etc.). Their research relies on an insightful longitudinal case study and offers advice on how to improve production ramp-up management.

More recently, Glock and Grosse $(2015)^{18}$ review different decision-support models for production ramp-up management and identify several basic production planning problems such as capacity expansion, product development, and learning aspects. The authors found that most of the existing research works address a single production planning problem while production ramp-up complexity suggests that these problems are interrelated. In fact, the authors underline the potential of simulation as a means to address the production ramp-up process complexity and as a way to move beyond analytical models prevailing so far in the literature. In particular, multi-agent simulation allows us to capture qualitative properties of the system under study based on agents' behaviours and interaction. Quantitative properties can be captured through mathematical modelling. ${ }^{23,24,25}$ Therefore, multi-agent simulation and mathematical modelling complete each other towards supporting ramp-up management decisions. The challenge remains how to unleash their synergies to model complex systems at large and to address ramp-up management in multivariant production, in the particular context of this research.

This paper addresses the problem of how best to support decision-making during the planning stage of ramp-up as a means to the effective management of a diverse product portfolio. The proposed approach leverages an agent-based simulation and performance indicators to provide an economic assessment of different ramp-up management strategies. The new model addresses the alignment of production with the customized demand during the ramp-up phase and applies this to multivariant production. 


\section{Production Ramp-Up Management and MAS Simulation}

\subsection{MAS Potential for Ramp-Up Management}

MAS paradigm has been applied to different problems in the areas of systems engineering and management and operations management. ${ }^{25,25^{26,27}}$ During the last few years, concepts such "holonic manufacturing systems" and "intelligent control of distributed systems" have relied heavily on $M A S{ }^{32,33,34}$ A holonic architecture of manufacturing systems is characterized by the autonomy of actions and decisions of system entities, leading to higher system flexibility. This trend is heightened by the increase in product variety and the emergence of small-lot-size production which requires a robust system with a higher degree of flexibility and reconfigurability. ${ }^{32,35,36}$

The wide application areas of MAS are partially due to the intrinsic characteristics of "agents" regardless of the development approach and implementation environment. In fact, autonomy and interaction are key features of an agent, which can be defined as a "computer system located in an environment that operates autonomously and flexibly to achieve the objectives for which it was designed". ${ }^{28}$ These characteristics are very consistent with the requirements of a production rampup phase, which is characterized by high uncertainty and the need for system adaptability. Additionally, while single agents are autonomous entities, they usually operate in a "society of agents" to achieve a general objective. ${ }^{29} 29,30,31$ This feature brings $M A S$ forth as a promising solution approach for complex problems by deconstructing the system or problem into independent sub-systems. Subsequently, $M A S$ exhibits a real potential to address inherently complex problems in high variety and small-lot size production environments. For instance, $M A S$ is a promising way to represent modular and smart systems, which are a key feature of high variety production environments.

\subsection{MAS Applications in Ramp-Up Management}

Vrba et al. (2015) ${ }^{37}$ introduce a scheduling model implemented using agents for the ramp-up of aircraft manufacturing. The proposal relies on mathematical modelling and the resulting model is integrated within an overall Information Technology (IT) architecture. Doltsinis et al. (2020) ${ }^{36}$ develop a decision-support system fostering the learning process as a means to improve the 
production ramp-up process. The proposal focuses on a task-level perspective and involves human agents in a production ramp-up learning process. Zhang et al. (2016) ${ }^{33}$ develop an agent-based architecture for intelligent manufacturing control focusing on the integration of the cyber- and physical- systems using RFID technology. The intelligent control framework is intended to improve flexibility and adaptability through a more efficient real-time production planning and control. In the same vein, Cupek et al. (2016) ${ }^{35}$ develop a holon-based model which allows a Manufacturing Execution System (MES) to be adapted to small-series production. The model is implemented into an IT architecture allowing for local optimization of the distributed production. The need for proactively dealing with changes and uncertainty has been further addressed by Rodrigues et al. (2018) ${ }^{34}$, who develop a MAS for service reconfiguration in manufacturing systems. The system considers factors such as production changeover. Authors such as Antzoulatos et al. (2014) ${ }^{38}$ and Kovalenko et al. (2019) ${ }^{39}$ develop multi-agent software architectures to support quick reconfiguration of production systems. MAS use in smart manufacturing systems also started to enhance additional dimensions such as sustainability and service. ${ }^{40}$ Research efforts conducted within European projects have resulted in relevant tools such as conceptual models and ontologies for production management using MAS. ${ }^{41,42}$ These efforts have also contributed to building a bridge to service-oriented smart manufacturing systems. ${ }^{43}$

In conclusion, the literature shows a discrepancy between the underlying pressing problem demanding new, innovative solutions to support production ramp-up for multi-variant, masscustomized products, and the available research. While the reported research and directions are motivated by the broader, customer-driven trends of mass-customization and multi-variant production, most of the literature is still focussing on developing solutions to optimize stable production rather than an agile production ramp-up phase. The selected research that addresses issues around production ramp-up is mainly focussed on the operational level, neglecting other key tactical and strategic perspectives including the economic outcome of different production rampup strategies. The significant amount of uncertainty underlying the ramp-up phase requires appropriate frameworks and operational tools to mitigate risk and enlighten decision-makers. ${ }^{44}$ 


\section{An MAS for Supporting a Production Ramp-Up Strategy Selection}

\subsection{Methodology and Background}

MAS development relies on 1) GAIA methodology supporting complex systems understanding and modelling, 2) Unified Modelling Language (UML) and platform-specific requirements to develop more detailed models and ease MAS implementation, and 3) Mathematical modelling to implement different production management strategies. ${ }^{45,46,47}$

GAIA inherits from the terminology of object-oriented design and analysis which ensures flexibility and fosters modular and rapid model development. ${ }^{48}$ It has been applied to address various problems in production and supply chain areas. ${ }^{48,49}$ Figure 2 shows GAIA basic models which guide system analysis and design as key steps in understanding and modelling complex systems. ${ }^{45}$ Requirements statement is an input for the GAIA methodology that represents the purpose of the system to be analysed and designed.

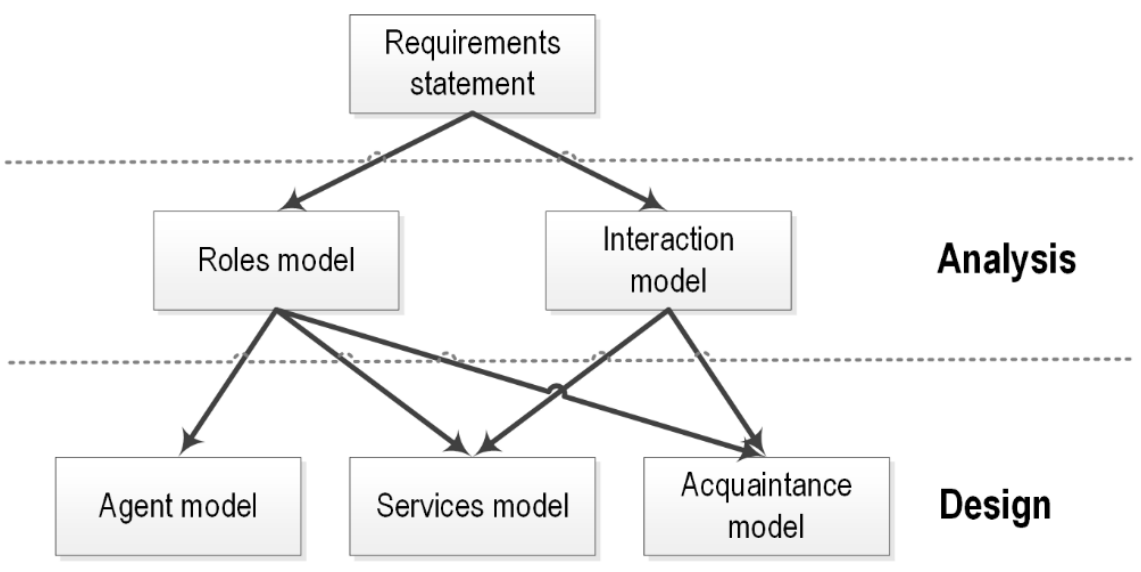

Figure 2. GAIA Basic Steps ${ }^{45}$

The analysis stage aims to build an understanding of the system organisation defined by a set of interacting roles. Two basic models are used in this step, Role Model and Interaction Model. The Role Model is defined by the following attributes: responsibilities, permissions, activities, and protocols. ${ }^{45}$ Responsibilities determine the functions associated with a role. Responsibilities could involve two different properties, Liveness and Safety. Liveness properties refer to desired states of affairs while Safety properties design the constraints that should be respected. Permissions refer to 
the rights of a role supporting the realization of its responsibilities. Activities are private actions associated with a role. Lastly, Protocols describe the way a given role interacts with other roles. The Interaction Model captures the interplay between roles to ensure system functioning. It consists of a set of protocols that are institutionalized patterns of interaction. A protocol definition relies on the following attributes: Purpose (i.e., nature of the interaction), Initiator (i.e., the responsible role for starting the interaction), Responder (i.e., the role with which the initiator interacts), Inputs (i.e., information used by the initiator), Outputs (i.e., the information supplied to/by the protocol responder), and Processing (i.e., possible initiator processing during the interaction).

The design stage is concerned with transforming the abstract models developed during the analysis stage to support technical implementation. The basic three GAIA model types used for this step are the Agent Model, Service Model, and Acquaintance Model. Agent Model defines agent types and agent instances that will release them at run time. To optimise system design, several agent roles can be aggregated into a single type. Service Model specifies the functions associated with each agent role known as services, which represent single and coherent activity blocks. Services' inputs and outputs are derived from the protocols. The Acquaintance Model defines the communication links between agent types and can be derived from roles and protocols. This model, however, does not specify further details such as message content.

Since GAIA methodology is not primarily concerned with the implementation, the design stage is broken into three steps, Design I, Design II, and Implementation. Design I follows GAIA logic and extends MAS using UML class and sequence diagrams. Design II specifies the mathematical models defining ramp-up strategies. Implementation integrates the specific requirements of the MAS development platform to ease the implementation. These three complementary and logically linked steps further support the reduction of the abstraction level by defining how an agent society will cooperate and what is needed for each individual agent in such a cooperation process.

In the following section, the development of the MAS is detailed, highlighting the requirements statement and MAS analysis (§4.2), MAS design I (§4.3), MAS design II (§4.4), and MAS implementation (\$4.5). 


\subsection{Requirements Statement and MAS Analysis}

The MAS is developed to support the assessment of ramp-up management strategies, in particular in a multi-variant production context. The scope of the research involves a single echelon supply chain adopting a Make-to-Order production management strategy. The focus is on a focal company dealing with a given market demand and being supplied with a set of components and raw material by its suppliers. Therefore, the internal organisation of the customers and the suppliers is out of this research's scope. Similarly, a limited number of agent types will be required which are also consistent with the scope of the GAIA methodology.

The roles required for the purpose of the current MAS development are Customer, Company, Production, Supplier, and Performance (see Table 1). The roles were selected and defined to represent a standard supply chain model in line with previous research works, building on the Supply Chain Operations Reference (SCOR) model. ${ }^{30}$ The SCOR model is widely accepted in industry and academia and relies on a standardized representation of the supply chain reinforcing replicability, performance measurement, and improvement. The protocols and activities shown in Table 1 are inspired by the SCOR model (Levels 1 and 2 from the SCOR model hierarchy). Unlike existing works dealing rather with shop-floor level, ${ }^{4,35,36}$ the current model aims to consider a broader perspective of the focal company covering its operations. The Company role is concerned with managing operations to fulfil customer orders, this involves both planning and execution activities. Production role is in charge of executing production orders and managing related workload and order queue. Supplier is introduced basically as a means to model the supply process as part of the company's operations. Table 1 shows Liveness responsibilities associated with agents' roles (last column). The supply and delivery activities are associated with the Company role (see Table 1). It assumes that the transportation activities from Suppliers to Company are managed by the Suppliers. The transportation to the Customer as part of the Delivery activities is ensured by the Company. Performance is responsible for recording the performance data and calculating indicators.

A particular and original feature of the proposed model is the structured "order management process". Orders are generated based on available configuration options of the product range based on the Customer role. Afterwards, the Company role processes the orders to determine whether the requested configuration is within the offered range and then plans material requirements. As such, 
orders are not considered as black boxes and the variety impact can be analysed more consistently and reliably. Additionally, both the product mix and order generation can be flexibly configured to reflect different strategies (e.g., low-mix, high-mix, etc.) and situations (e.g., demand volume, mean, deviance, etc.) within both ramp-up and stable production phases.

Table 1. MAS Roles Model and Roles Definition

\begin{tabular}{|c|c|c|c|}
\hline $\begin{array}{c}\text { Role } \\
\text { Schema }\end{array}$ & Description & $\begin{array}{l}\text { Activities } \\
\text { Protocols }\end{array}$ & $\begin{array}{c}\text { Responsibilities } \\
\text { Permissions }\end{array}$ \\
\hline Customer & \begin{tabular}{|c|} 
Generate order \\
according to a given \\
probability distribution
\end{tabular} & $\begin{array}{c}\text { Generate order } \\
\text { Send order } \\
\text { Order Request Generation }\end{array}$ & Customer $=($ Generate order, Send order $)$ \\
\hline Company & $\begin{array}{l}\text { Manage operations to } \\
\text { fulfil customer orders }\end{array}$ & $\begin{array}{c}\text { Receive customer order } \\
\text { Process order } \\
\text { Manage inventory } \\
\text { Prepare order } \\
\text { Deliver order } \\
\text { Customer Order Processing } \\
\text { Supply Order Request } \\
\text { Production Order Request }\end{array}$ & $\begin{array}{c}\text { Company }=\text { (Receive customer order, Process order, } \\
\text { Manage inventory, Prepare order, Deliver order }) \\
\text { Read Customer order }\end{array}$ \\
\hline Production & $\begin{array}{l}\text { Execute production } \\
\text { activities }\end{array}$ & $\begin{array}{l}\text { Receive production order } \\
\text { Update production load } \\
\text { Deliver production order } \\
\text { Production Informing Company }\end{array}$ & $\begin{array}{c}\text { Execute production activities }=\text { (Receive production } \\
\text { order, Update production load, Deliver production } \\
\text { order) } \\
\text { Read Production order }\end{array}$ \\
\hline Performance & $\begin{array}{l}\text { Record performance } \\
\text { indicators }\end{array}$ & $\begin{array}{l}\text { Update and record cumulative } \\
\text { performance indicators } \\
\text { Update and record performance } \\
\text { indicators }\end{array}$ & $\begin{array}{c}\text { Track and update performance indicators }=\text { (Update and } \\
\text { record cumulative performance indicators, Update and } \\
\text { record performance indicators) } \\
\text { Read and update indicators }\end{array}$ \\
\hline Supplier & $\begin{array}{c}\text { Supply focal company } \\
\text { with raw material and } \\
\text { components }\end{array}$ & $\begin{array}{l}\text { Receive Company order } \\
\text { Process Company order } \\
\text { Deliver Company order } \\
\text { Supply Order Fulfilment } \\
\text { Supply Informing Company }\end{array}$ & $\begin{array}{c}\text { Supplier }=(\text { Receive Company order } \\
\text { Process Company order } \\
\text { Deliver Company order }) \\
\text { Read Company order }\end{array}$ \\
\hline
\end{tabular}

Figure 3 shows the interaction model highlighting the protocols enabling order management by the developed MAS. This model is focused on the purpose of the interactions happening across the MAS, rather than the sequencing of these interactions. The eight basic protocols comprising the interaction model are Order Request Generation, allowing to simulate the demand of a given product mix, Customer Order Processing, allowing to process the orders coming from the customer and to plan material requirement, Supply Order Fulfilment and Production Order Request, generated based on material requirement planning, Production Informing Company and Supply Informing Company, to inform the company of order reception, and Customer Order Fulfilment, referring to order delivery to the final customer. 

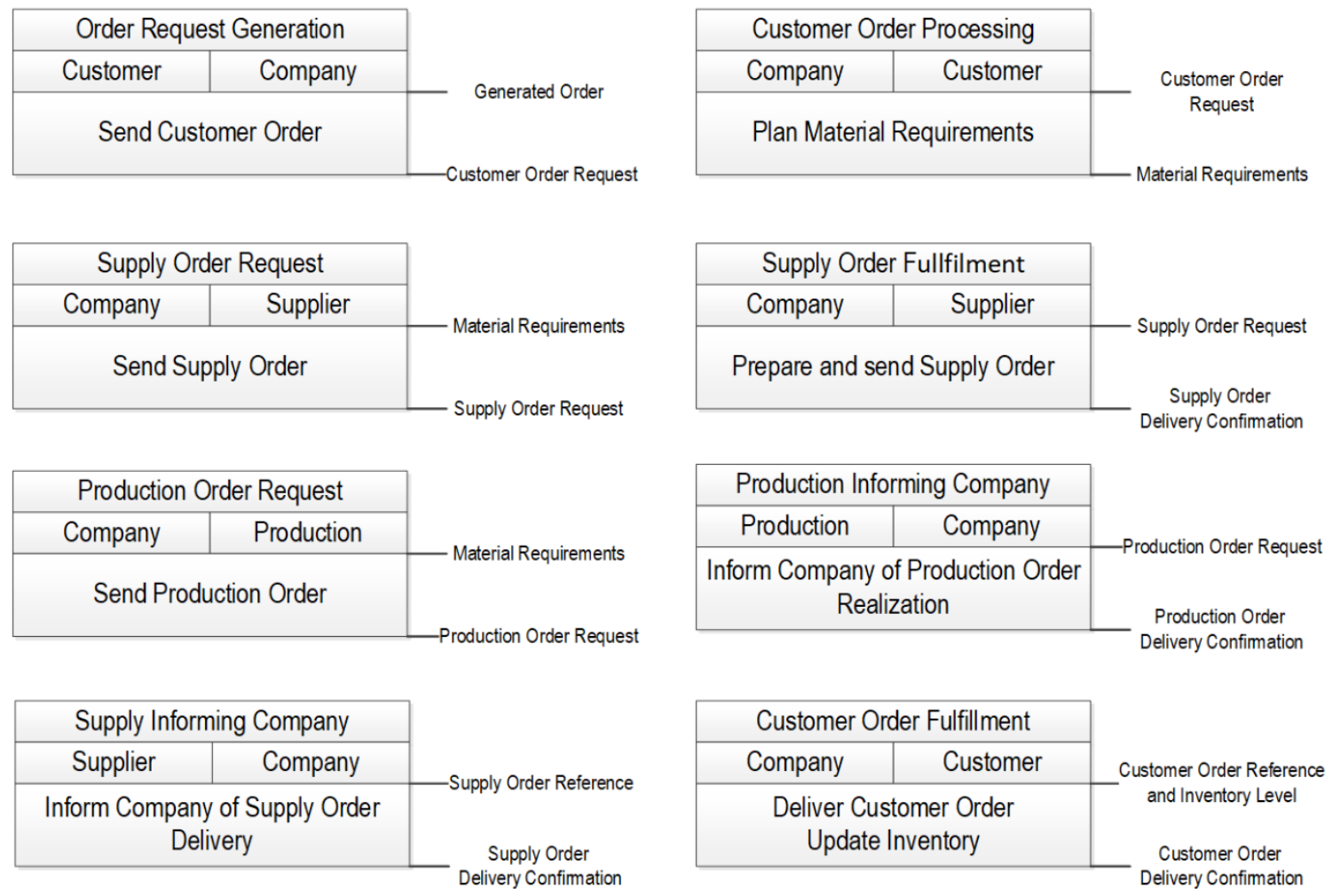

Figure 3. MAS Interaction Model

\subsection{MAS Design I}

Following GAIA methodology, Agent Model, Acquaintance Model and Service Model were developed to provide more detailed modelling of the system to ease its implementation. These models are represented respectively in Figure 4a, Figure 4b, and Table 2. To further define how an agent performs and cooperates, a UML class diagram and sequence diagram are used.

Figure 5 reports on the sequence diagram, highlighting the general sequence of order management. The typical operation scheme starts with the company receiving an order from the customer. The scenario represented in Figure 5 illustrates a typical order fulfilment process and assumes the order management is automated and that there is no bullwhip effect. ${ }^{50}$ This is consistent with the current scope of the model and to start to build a foundation for ramp-up management of multi-variant production. In the current version of the proposed MAS, the Performance role is limited to data collection, as well as calculation and update of performance indicators. Therefore the Performance role is not represented in the sequence diagram depicted in Figure 5. However, 


\section{the authors assume it is preferred to represent it as an agent rather than a program since it}

can be supplemented with further performance monitoring functions. For instance, deriving recommendations for the indicators and sending them to the Company agent.

(a)

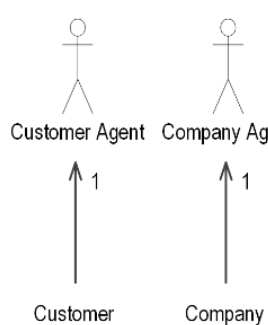

Customer

Company
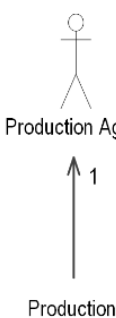
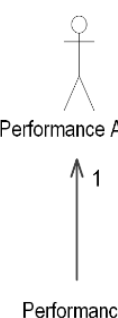

Figure 4. MAS Agent Model and Acquaintance Model

Table 2. MAS Service Model

\begin{tabular}{|c|c|c|c|c|c|}
\hline Agent & Service & Inputs & Outputs & Pre-condition & Post-condition \\
\hline \multirow{2}{*}{ Customer } & Generate order & $\begin{array}{l}\text { Product portfolio } \\
\text { Demand profile }\end{array}$ & Customer order & & \\
\hline & Send order & $\begin{array}{c}\text { Customer order } \\
\text { Company Identifier } \\
\end{array}$ & Order sent & & $\begin{array}{c}\text { Company informed } \\
\text { of the order }\end{array}$ \\
\hline \multirow{5}{*}{ Company } & Receive customer order & Customer order & Order recorded & Order queue available & $\begin{array}{c}\text { Order queue } \\
\text { updated }\end{array}$ \\
\hline & Process order & Recorded order & Material requirements & & \\
\hline & Manage inventory & $\begin{array}{l}\text { Material } \\
\text { requirements }\end{array}$ & $\begin{array}{l}\text { Updated material } \\
\text { requirements } \\
\text { Supply order } \\
\end{array}$ & & $\begin{array}{c}\text { Performance } \\
\text { indicators updated }\end{array}$ \\
\hline & Prepare order & $\begin{array}{l}\text { Production order } \\
\text { Supply order }\end{array}$ & Inventory level updated & $\begin{array}{l}\text { Production informed } \\
\text { Supplier informed }\end{array}$ & $\begin{array}{c}\text { Performance } \\
\text { indicators updated }\end{array}$ \\
\hline & Deliver order & $\begin{array}{l}\text { Customer order } \\
\text { Inventory level }\end{array}$ & Order delivered & Stock available & $\begin{array}{c}\text { Stock updated } \\
\text { Performance } \\
\text { indicators updated } \\
\end{array}$ \\
\hline \multirow{3}{*}{ Production } & $\begin{array}{l}\text { Receive production } \\
\text { order }\end{array}$ & Production order & Order recorded & & \\
\hline & \begin{tabular}{|l|} 
Update production load \\
\end{tabular} & Recorded order & Updated production load & & \\
\hline & \begin{tabular}{|c|}
$\begin{array}{c}\text { Deliver production } \\
\text { order }\end{array}$ \\
\end{tabular} & $\begin{array}{l}\text { Production order } \\
\text { Inventory level }\end{array}$ & Inventory level updated & & $\begin{array}{c}\text { Performance } \\
\text { indicators updated }\end{array}$ \\
\hline \multirow[b]{2}{*}{ Performance } & $\begin{array}{c}\text { Update and record } \\
\text { cumulative performance } \\
\text { indicators }\end{array}$ & $\begin{array}{c}\text { Current } \\
\text { performance } \\
\text { indicator values }\end{array}$ & $\begin{array}{l}\text { Updated performance } \\
\text { indicator values }\end{array}$ & Data record time & \\
\hline & $\begin{array}{c}\text { Update and record } \\
\text { performance indicators }\end{array}$ & $\begin{array}{c}\text { Current time } \\
\text { Current } \\
\text { performance } \\
\text { indicator values }\end{array}$ & $\begin{array}{l}\text { Recorded performance } \\
\text { indicator values }\end{array}$ & & \\
\hline \multirow{3}{*}{ Supplier } & Receive Company order & Company order & Company order recorded & & \\
\hline & Process Company order & \begin{tabular}{|c|} 
Recorded company \\
order
\end{tabular} & Processed order & & \\
\hline & Deliver Company order & Processed order & Order sent & & $\begin{array}{c}\text { Company informed } \\
\text { of the order }\end{array}$ \\
\hline
\end{tabular}

In the represented scenario, both Customer and Company agents are "active" before sending and receiving an order. In fact, before sending the order, the customer needs to generate a product 
reference and quantity to include in the order. Both Company and Customer require initialization to load master data about demand and production. The company agent receives the order and then calculates material requirements according to which it sends supply orders and production orders to supply and production agents, respectively. The activity of the production and supply agents starts upon receiving the company order. Upon receiving the required material and production order, the company proceeds with preparing the order and delivering it to the final customer.

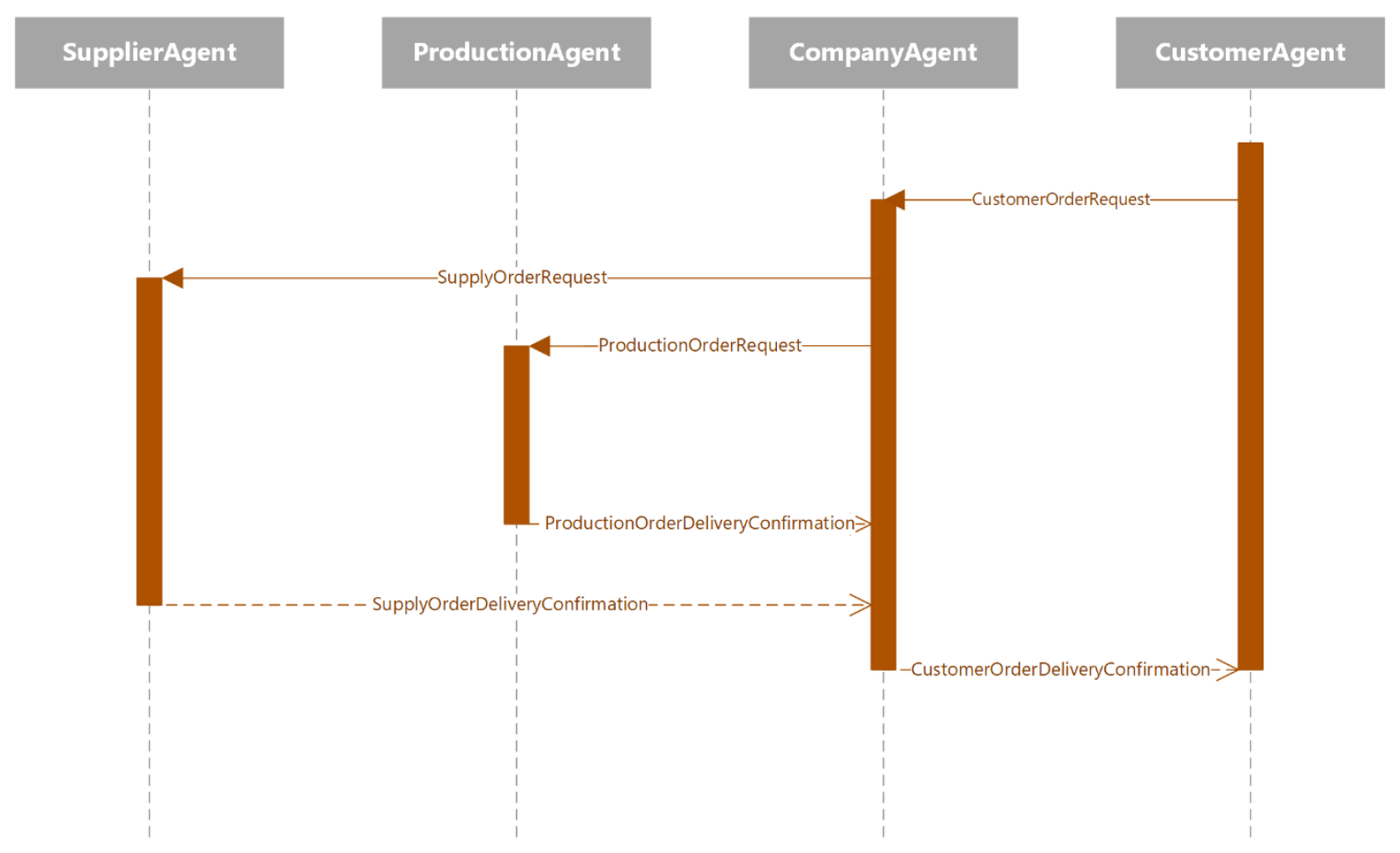

Figure 5. MAS Model Sequence Diagram

\subsection{MAS Design II}

This step aims to specify the detailed operations of the agents belonging to the MAS under development using a class diagram and mathematical modelling. A summary of agents' operations derived from the Service Model is given by the UML class diagram shown in Figure 6. The mathematical model allows for implementing different ramp-up strategies. Examples of these include high-volume low-mix (HVLM) and low-volume high-mix (LVHM). The choice of strategy depends on product variety and complexity, for instance, an HVLM may fit relatively standard products while more complex and highly customized products may require an LVHM strategy. A given strategy can be translated into a production plan described through the matrix $S$ representing 
the planned production volumes of each product $i$ at each planning period $t, p_{i}^{t}$, where $M$ refers to the total number of different products included in a Company's portfolio and $T$ is the total number of planning periods considered for the ramp-up phase (6 to 24 months to remain consistent with ramp-up context).

$$
S=\left(p_{i}^{t}\right)_{i=1 . . M, t=1 \ldots T}
$$

$p_{i}^{t}$ is calculated based on the share of product $i$ in the product mix at period $t, s_{i}^{t}$, and the total planned aggregate production capacity during period $t, P^{t}$ (Eq. 2). The effective volume produced from $i$ at $t, e_{i}^{t}$, is an adjustment of the planned volume $p_{i}^{t}$ based on customer orders received during period $t$ (Eq. 3). The determination of $P^{t}$ is derived from the demand forecast $F^{t}$ and adjusted to mitigate production disturbances. However, demand forecast and its impact on aggregate capacity planning is considered out of the scope of the model, therefore the model input is assumed to be known, $P^{t} \forall t \in\{1, \ldots, T\}$. $s_{i}^{t}$ depends on the complexity of product $i, C_{i}$, planned aggregate production capacity during the period $t, P^{t}$, and the period $t$ (Eq. 4). $s_{i}^{t}$ is valued by decisionmakers considering the importance and intensity of these factors in the ramp-up context. The complexity of the product and its process adds to the complexity and uncertainty of the ramp-up phase. The period $t$ allows possible adjustment of the planned production volumes according to the positioning within the ramp-up phase, e.g., beginning of the ramp-up (small volumes to partially meet the total demand), end of the ramp-up (stable production and high volumes to meet full demand).

$$
\begin{gathered}
p_{i}^{t}=s_{i}^{t} \times P^{t} \\
e_{i}^{t}=\sum_{i} d_{i}^{t} \mid e_{i}^{t} \leq p_{i}^{t} \\
s_{i}^{t}=g\left(C_{i}, P^{t}, t\right)
\end{gathered}
$$

Performance agent collects performance data at predefined points of time which can be defined by the developer or user. Total cost is calculated incrementally in a two-step procedure: first, summing up supply $\operatorname{cost} c_{S}^{t}$, production $\operatorname{cost} c_{P}^{t}$, delivery $\operatorname{cost} c_{D}^{t}$, and inventory holding $\operatorname{cost} c_{I}^{t}$, captured at recording period $t$ (Eq. 5), and the second, updating total $\operatorname{cost} c_{T}^{t}$ (Eq. 6).

$$
c^{t}=c_{S}^{t}+c_{P}^{t}+c_{D}^{t}+c_{I}^{t}
$$




$$
c_{T}^{t}=c_{T}^{t-1}+c^{t}
$$

Average cost and lead-time are incrementally updated according to Equations 7 and 8, respectively. This allows us to keep track of the trend within these indicators.

$$
\begin{gathered}
c_{A}^{t}=\frac{c_{A}^{t-1}+c^{t}}{2} \\
l_{A}^{i}=\frac{l_{A}^{i-1}+l^{t}}{2}
\end{gathered}
$$

Sales associated with a given order $s_{t}$, equal the simple product of unit selling price of the variant $v$, denoted as $s_{t}^{v}$ and the quantity per order denoted as $q_{t}^{v}$ (Eq. 9). Sales turnover $s_{T}^{t}$ is updated incrementally by summing sales per orders (Eq. 10).

$$
\begin{gathered}
s^{t}=s_{t}^{v} \times q_{t}^{v} \\
s_{T}^{t}=s_{T}^{t-1}+s^{t}
\end{gathered}
$$

Overall, the model for calculating indicators is purposefully simple as the focus of the current research is on the overall operations of the company rather than sophisticated models for specific activities at the shop-floor level. The model also covers a revenue perspective which is relevant to variety and ramp-up management as both tactical and strategic decisions depends not only on costs but also on expected revenues. In this sense, the model is complementary with existing research proposing structured approaches to address specifically detailed activities and focusing on operational performance.

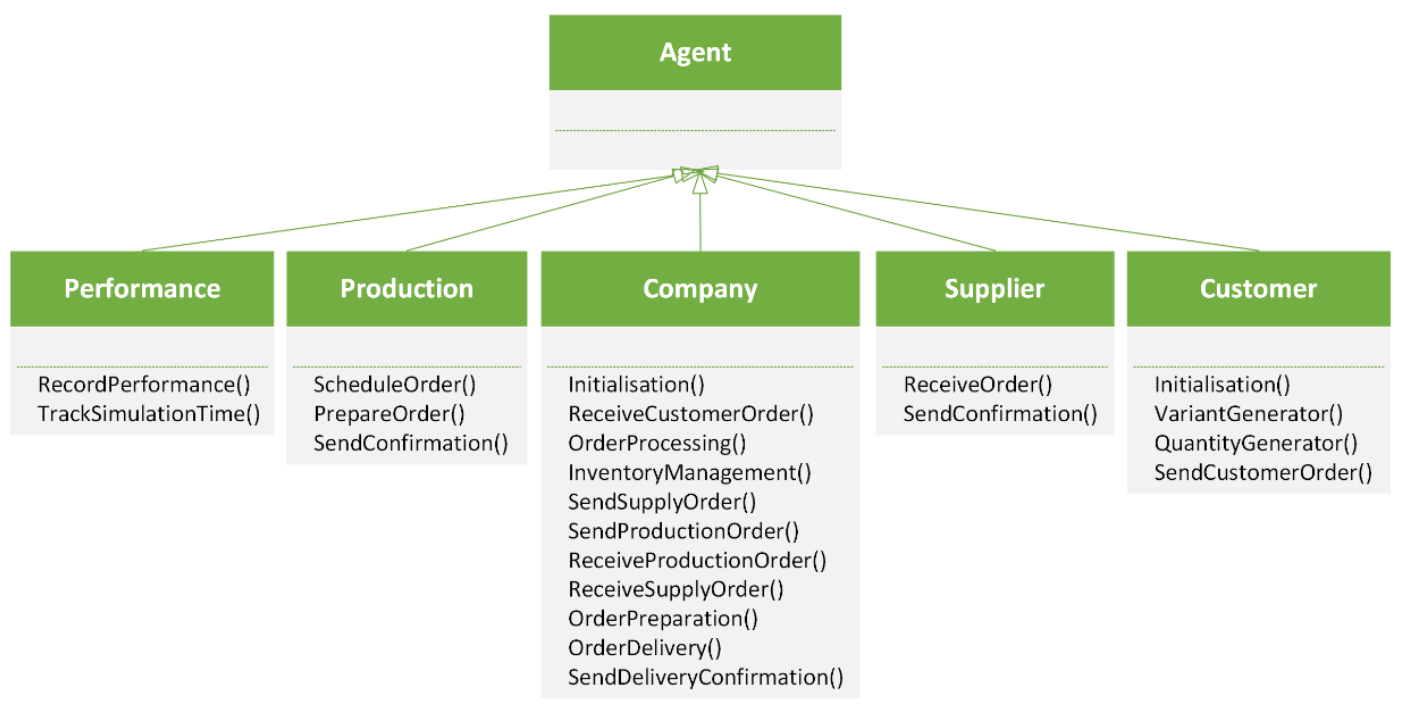

Figure 6. MAS Model Class Diagram 


\subsection{MAS Implementation}

This step aims to support the implementation of the MAS by integrating requirements from the MAS development platform. Figure 6 reports on main agents' operations derived from the activities described in the MAS analysis step. The development of the agent model was carried out to use the JADE (Java Agent Development Environment) platform for the implementation. JADE is a software framework supporting the development of agent applications consistent with the FIPA (Foundation for Intelligent Physical Agents) specifications for interoperable intelligent multiagents. ${ }^{51}$ Although several other commercial and open-source frameworks have been developed since then, JADE is still being widely used for several reasons. For instance, this open-source project offers a high degree of flexibility during the development of agents and MAS. Additionally, being consistent with FIPA standards plays a major role in the framework acceptance among users and communities. These features are among the drivers of the extensive use of JADE in the systems engineering and management, and operations management fields. ${ }^{52,53}$ Within JADE, messages are managed using the Agent Communication Language (ACL), message sending and reception is dealt with using SimpleBehaviour. Request and Confirm performatives are assigned to ordering and delivery confirmation messages, respectively.

The main operations associated with the Customer agent relate to initialisation, order generation, and sending to the company. Customer operation is implemented through a SequentialBehviour comprised of an OneShotBehaviour for the initialisation and a TickerBehaviour for order generation. Initialisation allows, for instance, Customer agent to load data about the Bill-OfMaterial and possible product configurations as well as information about order arrival rate and quantities per order. Order generation refers to generating orders as per the initialized data specification. This allows for high flexibility in simulating various scenarios and strategies. Supplier agent has a limited set of operations consisting of receiving Company orders and sending the delivery confirmation after a given period of time.

The Company agent has several operations, as shown in Figure 6, given its key role within the MAS. The initialisation allows the master data about production and product portfolio to be uploaded. These data include available variants (i.e., configurations), their Bill-Of-Material, process data, and other technical data. While initialisation is ensured by OneShotBehaviour, the remaining operations are implemented through a ParallelBehaviour. Order Processing is 
implemented through a CyclicBehaviour making it possible to continuously check incoming customer order queues. Order Processing checks the feasibility of the order and then calculates material requirements. The other operations, except sending and receiving messages, are implemented through TickerBehaviours. Production agent operations are limited to scheduling the order, preparing the order, and confirming to Company. Orders are scheduled using a simple First In, First Out (FIFO) rule upon updating the production load. The updated production load is used afterwards in a WakerBehaviour representing the production time. A confirmation is sent to the Company upon completing the production order. Performance agent has access to cost, lead-time, and inventory level indicators. Its main operations include recording performance and tracking simulation time. Recording performance is implemented through a TickerBehaviour allowing performance indicator values to be recorded at given points of simulated time. The Performance agent is responsible for terminating the simulation according to the pre-specified simulation horizon.

\section{Illustrative Case in the Furniture Industry Sector}

In this section, we present a prototypical implementation and evaluation of the developed MAS in the form of a case study. The objective is to illustrate the applicability of the developed MAS in industry.

\subsection{Case Company Selection and Overview}

The case study was selected based on its fit with the requirements of the developed MAS methodology and the possibility of evaluating the developed MAS. In essence, the case study aligns with the proposed MAS objective to support the assessment of ramp-up management strategies.

To remain consistent with the scope of the current research, a multi-variant production context is required as part of the case study. Priority target companies are SMEs (Small and Medium-sized Enterprises) which traditionally require more decision-making support on the strategic and tactical levels. The furniture industry is among the suitable candidates that meet these requirements. This sector is growing due to favourable market trends and prevailing lifestyles. In the kitchen furniture sector, for instance, customization and high variety are two main features that customers demand, 
and many manufacturers are offering today. This presents a major challenge for companies to achieve their objectives concerning "time-to-market" and "time-to-volume".

The selected case company for our study is a kitchen furniture manufacturing SME located in Europe that supplies a wide range of markets ranging from mid-range to luxury kitchens. With regards to their product offering, the basic components are the kitchen cabinets. Additional accessories are supplied by several partners of the company and generally do not pose a particular challenge with regards to ramp-up. Therefore, the focus of our study will be placed on the different variants of kitchen cabinets that are offered by the company. A competitive advantage of the selected case company is the opportunity to easily customize kitchen cabinet sizes.

The company's portfolio includes luxury and highly customized kitchens, produced on EngineerTo-Order (ETO) basis, and medium-range kitchens, produced on Make-To-Order (MTO) basis. The case study involves six variants of the kitchen cabinet with different sizes, belonging to medium range kitchens. This scope was purposefully defined in order to (i) show how a limited variety and complexity can impact the performance, and (ii) to keep the focus on the illustration of the assessment of production ramp-up management strategies.

Table 3 shows the list of variants and their selling prices - the variants are ordered according to their sizes, with five being the biggest cabinet model. Hourly production costs amount to approximately $€ 56$ while the cost per delivered order is about $€ 40$. The average lead-time (in days) of the production, delivery, and supply is about $0.04,1$ and 3 , respectively. Order costs amount to $€ 50$ and the stock holding ratio is about $3 \%$. In order not to lose focus, detailed parameters of inventory management will not be discussed. These parameters will not be changed among the simulation scenarios. The default demand profile follows a normal distribution with a mean of 10 and a deviation of 5 .

Table 3. Selling Prices

\begin{tabular}{|c|c|}
\hline Variant & Selling Price $(\boldsymbol{\epsilon})$ \\
\hline 0 & 550 \\
\hline 1 & 500 \\
\hline 2 & 350 \\
\hline 3 & 400 \\
\hline 4 & 600 \\
\hline 5 & 650 \\
\hline
\end{tabular}


The MAS was implemented in Java language using the eclipse platform with the JADE plugins. Simulations are run using an Intel Core i5, 2.30 GHz. A simulation run to simulate the supply chain for one year takes 20 seconds. A time conversion ratio is used to convert the real-time into system time (e.g., one day is equivalent to 40 milliseconds). The frequency for recording performance data by Performance agent is based on the conversion ratio (e.g., if performance data need to be recorded every week, then Performance agent will proceed with recording every 200 milliseconds (5 x 40 milliseconds, assuming that a week is five working days)). Figure 7 presents an excerpt of the sniffer agent which depicts interactions among Company, Customer, Production, and one supplier (Supplier14), during a short period of the simulation time.

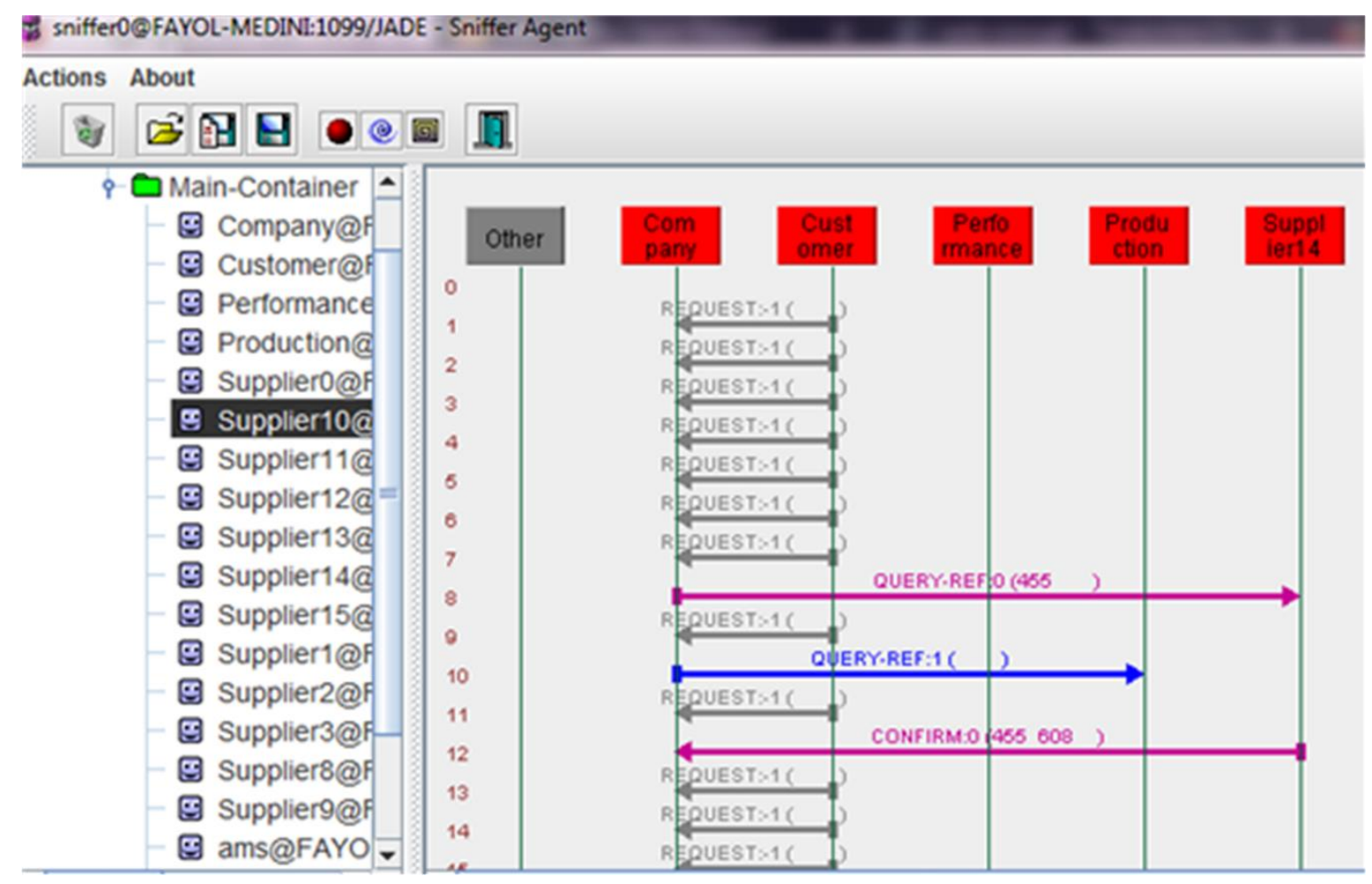

Figure 7. MAS Interaction During Run-time

\subsection{Simulation Results}

To support a production ramp-up management strategy selection, two basic scenarios were defined in the first place. The first scenario involves a High-Volume-Low-Mix (HVLM) strategy, and the second scenario represents a Low-Volume-High-Mix (LVHM) strategy. In the HVLM scenario, the offering consists of variants 2 and 3 which are the most standard and cost-efficient ones. 
Production is assumed to be equally shared between these variants. In the LVHM scenario, all variants are included in the offering with the following shares: $10 \%$ for each of variants 0 and 6 , and $20 \%$ for each of the other variants. In the second scenario, the total production is reduced in order to respect capacity constraints. This is because the average production lead-time increases by $20 \%$ when all variants are included in the offering. The idea is to evaluate different alternatives for the same total capacity. This is also in line with past research. ${ }^{53}$ Each of the two simulation runs covers a one-year horizon.

The results of the simulations are referred to by HVLM and LVHM in Figure 8. At first glance, it can be seen that the company could perform well when pursuing an HVLM strategy during the first year, with a higher sales turnover and $€ 236 \mathrm{~K}$ profit. By contrast, the LVHM strategy leads to lower sales turnover and lower profit, with a decrease of 10 to $20 \%$. This is down to the decrease in productivity given the higher complexity associated with variants $0,1,4$, and 5 which require a higher production lead time and induce higher costs.

It was decided to increase selling prices when adopting an LVHM strategy to be consistent with the prevailing logic in businesses offering higher variety and customization. This third simulation scenario is referred to as LVHM-P where selling prices are increased by $6 \%$. Figure 8 shows that although sales turnover is still lower than the HVLM scenario, the profit is $10 \%$ higher in this LVHM-P scenario. This indicates the relevance of pursuing an HVLM strategy while revising selling prices to enhance the economies of scope. This is evidenced further by the rate of return, which climbs from 0.54 in the HVLM scenario to 0.61 in LVHM-P. 


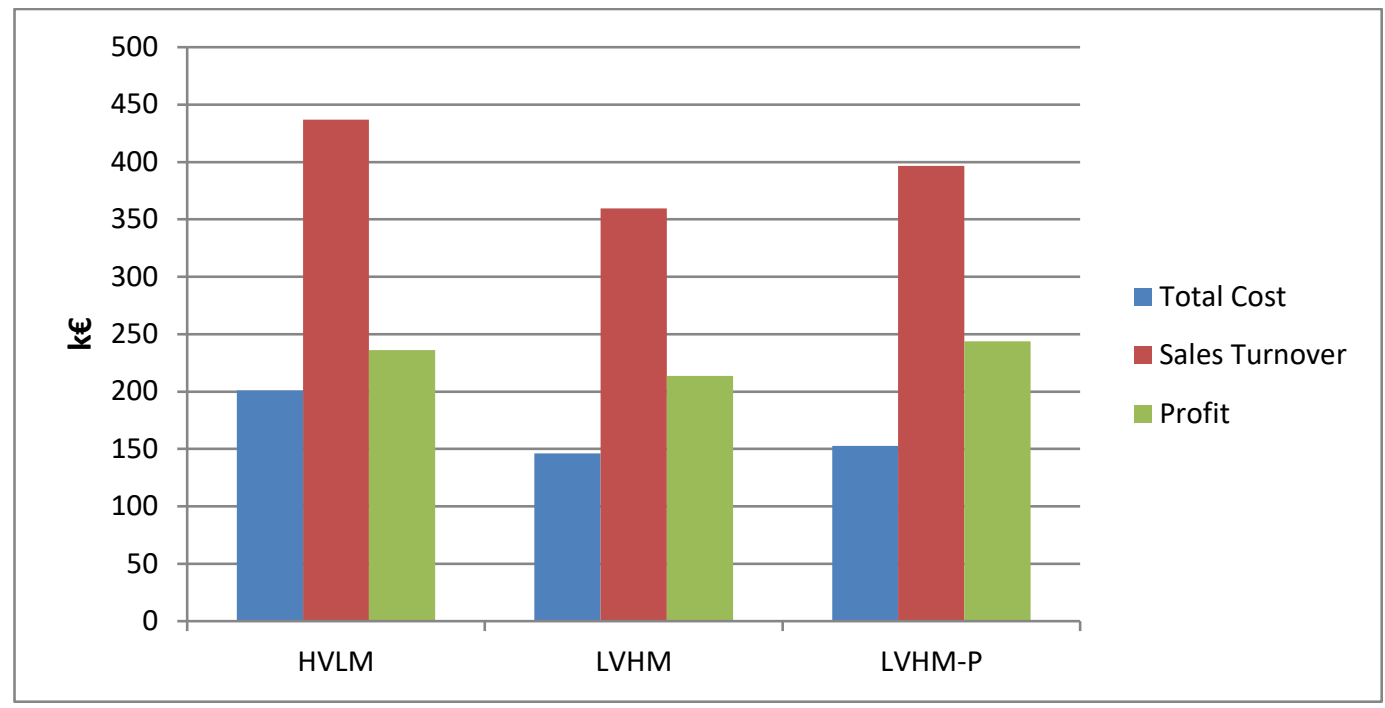

Figure 8. Economic Performance Results

While the case study informs the decision-maker about the expected costs and benefits of ramp-up strategies, no definite preference emerges immediately as to which strategy is more appropriate for a given scenario. In fact, the decision depends on several strategic, tactical, and operational factors. At a strategic level, whether the company enters the market as an innovator or achieves economies of scale through cost efficiency is a critical factor when selecting a suitable production ramp-up strategy. Furthermore, factors such as customer willingness to pay in case of increased prices, and the usefulness of offering variety, are to be taken into account. At the tactical and operational levels, the decision on which strategy to pursue depends on internal variety-induced complexity, and thus, on cost and lead-time, production capacity, resource availability, and human capital qualification, just to name a few. This finding aligns with past research highlighting that HVLM and LVHM are equally distributed among most companies' strategies.

\section{Discussion}

Today's manufacturer faces many challenges stemming from changing customer demands, such as the provision of personalized products with similar efficiency and time to market as mass-produced standard products. Providing support in addressing these challenges is of critical importance for many companies, especially SMEs. Ramp-up management is understood as a critical factor to meet this challenge as it focuses specifically on the transition phase from product development to stable 
production. In this regard, theoretically sound, yet practice-oriented interdisciplinary research is required to address these challenges holistically.

To address this research gap, our paper expands ramp-up management through frameworks relevant to the current business context, which is shaped by overwhelming complexity. In particular, this paper underlines the relevance of MAS to support decision making in rampup management. This is further reinforced by the conducted literature analysis and the presented illustrative case study. With the MAS design method detailed in Section 4 and illustrated by a case study, the paper lays a foundation for further investigation and development to unleash MAS' benefits for ramp-up management in multi-variant production environments. Compared to existing MAS applied to supply chain and operations management fields, the current proposal is consistent with the idea of aligning operations management with customer demands. This allows the integration not only of costs, but also potential profits associated with different ramp-up strategies in a holistic cost-benefit analysis. This idea is implemented in the MAS through an order management system based on a make-to-order strategy triggered by customer orders, which are generated according to different market scenarios. By doing so, it becomes possible to adapt ramp-up strategies to production capacity but also and most importantly to demand profiles (e.g., adapting production volumes and product mix). Furthermore, unlike most of the existing models, our proposed MAS relies on a comprehensive modelling of the supply chain spanning from end customer (order generation) to suppliers and detailing focal company operations. This limits, to some extent, simplified hypotheses, for example, concerning customer demand, delivery, supply activities, and so on, by extending the scope of the MAS, thus enhancing consistency of results. In order to enhance consistency, a performance role is designed and dedicated to tracking costs and sales data during a simulation. Finally, with our comprehensive yet simple model, we offer higher development flexibility and adaptation potential.

In line with the need for practical and reusable approaches, we elaborated on a design method for MAS, illustrate this with a simple MAS supporting ramp-up and operations management, and apply the MAS to a case company in the furniture sector, thereby offering valuable insights for decisionmakers in ramp-up management. As such, our paper contributes to decision-making in different ways: reusable MAS development method, reusable tool, and first validation. All three contributions support an ultimate managerial implication consisting of increased awareness of 
decision-makers of the ramp-up phase and enabling more informed decisions about ramp-up management. The proposed MAS informs decision-makers of potential impacts on the economic performance of different production ramp-up strategies, particularly when dealing with different product variants. The guidance of the decisions is two-fold; first, it allows to oversee different scenarios according to the company operation environment and internal policies, and second, it allows to fine-tune the production ramp-up strategy before its implementation, e.g., defining a range for selling prices, establishing the product mix, etc.

The MAS developed, presented, and tested in this paper helped to derive multiple valuable insights for the use of MAS in the field of ramp-up management and opened several new research directions. These can be categorized into MAS improvement and MAS extension. Regarding MAS improvement, three main areas were identified: risk management, interactions, and negotiation. In fact, all agents' behaviours can be supplemented with new functionalities, and more sophisticated mathematical models can be easily implemented. Yet, the main challenge involves the identification and modelling of risk factors. Furthermore, the interactions between the agents can be reinforced to enable fine-tuning of agents' strategies using indicators calculated during run-time (e.g., feedback loops between Performance agent and Company agent). Finally, agents' responsibilities can be expanded by implementing negotiation protocols at inter-enterprise and intra-enterprise levels. The negotiation has shown efficacy concerning performance improvement in several application contexts. ${ }^{30}$

Several perspectives can be identified regarding the MAS extension, such as addressing different production ramp-up stages and supporting the decision-making process on ramp-up curves. ${ }^{6} \mathrm{~A}$ potential benefit of this extension is the clear definition of how the product development overlaps with the production phase and, most importantly, how to progressively get to a stable production process reaching the production ceiling. Additionally, although capacity is taken into account in the current case study, the link between product variety-induced complexity and the overall production capacity is based on hypotheses. A potential future research perspective might involve modelling this link through analysing the impact of complexity on capacity planning.

Furthermore, based on current research, multi-variant production ramp-up management can be supported by two possible pathways using a MAS approach: The first pathway addresses production ramp-up management through a combined digital factory and Cyber-Physical 
Production System (CPPS) approach using smart data for production planning, control, and troubleshooting. This data-driven approach aligns with the Industry 4.0 paradigm and supports the development of more flexible and agile smart manufacturing systems. Examples might include software agents, represented by interoperable information systems, supporting the vertical and horizontal integration of information for production planning and control; machine agents, represented by smart machines, reducing downtime and troubleshooting efforts (e.g., time and other resources) due to their ability for self-diagnosis, self-calibration, and prognosis abilities. The second pathway addresses product variety management through a product-centric production control approach enabled by the product agents, represented by smart products, which shift production control from Programmable Logic Controllers (PLCs) to a service-oriented and decentralized production control system enabled by the digital memory of a smart product. This digital memory will store all the necessary information for the manufacturing of a specific product (e.g., pharmaceutical, aerospace, etc.), allowing the product agent to have full control of the individual product's manufacturing process. The product agent will communicate autonomously with the different smart manufacturing systems' resources in the production line, named machinery agents, to negotiate its production plan in response to the delivery time agreed with the customer. A product-centric production control approach can then increase the flexibility of the smart manufacturing system for small production runs and mass-customized and/or mass-personalized products. This trend can benefit from the synergies with Industry 4.0 tools such as 3D printing and ultimately support a more efficient ramp-up management.

\section{Conclusions}

Industry 4.0 is reviving several concepts and research topics by integrating established and new methods and technologies in ways previously unheard of. Therefore, new opportunities for improving a firm's agility within its rapidly changing environment are being uncovered. More specifically, tools and methods for managing product/production variety and ramp-up reached a ceiling at some point in the past. The appealing concept of smart manufacturing systems within the set of Industry 4.0 tools is opening up new perspectives to efficiently meet customized demands with small-lot-size production. For instance, MAS exhibits the potential to easily and smoothly integrate intelligence embedded in software agents into physical systems while taking advantage of sensing and data processing technologies. 
This paper supports this momentum through a twofold structured design method and a MAS that supports the definition and fine-tuning of a robust ramp-up management strategy. The design method is detailed and illustrated through a $M A S$ to facilitate transparency and reusability. The newly designed and developed $M A S$ features high flexibility through implementing and adapting pre-defined behaviours. The results from the illustrative case study indicate its relevance to decision-makers during the ramp-up phase.

However, these opportunities come also with challenges, such as the complexity of projects in this area. In fact, cross-disciplinarity is a key requirement for a team that wishes to integrate different methods and technologies, for example, $M A S$, production management, product and process design. Therefore, it is not just different skills that are required, but most importantly, multi-skills should be acquired through innovative and better-adapted practices such as blended learning. Moving forward, the proposed approach needs to be tested in more complex supply chain scenarios to better understand the challenges of operationalizing it in industry and the limitations of the simulation when faced with a more complex scenario.

\section{Acknowledgement}

This work is partly supported by the German-French Academy for the Industry of the Future through RAMP-UP Seed project and by Face Foundation through SUSTAIN project.

\section{References}

1. WMF - World Manufacturing Forum, World Manufacturing Forum 2018 Report Recommendations for the Future of Manufacturing. World Manufacturing Foundation, 2018, https://perma.cc/DNY3-TWMP.

2. Lanza, G., Sauer, A., "Simulation of personnel requirements during production ramp-up", Prod. Eng., vol. 6, pp. 395-402, 2012, https://perma.cc/YL82-H73Z.

3. M. Colledani, T. Tolio, and A. Yemane, "Production quality improvement during manufacturing systems ramp-up," CIRP J. Manuf. Sci. Technol., vol. 23, pp. 197-206, 2018, https://perma.cc/DQS2-4ZGG.

4. S. Doltsinis, P. Ferreira, and N. Lohse, "An MDP model-based reinforcement learning approach for production station ramp-up optimization: Q-learning analysis," IEEE Trans. Syst. Man, Cybern. Syst., vol. 44, no. 9, pp. 1125-1138, 2014, https://perma.cc/RT7Y-3D7J.

5. U. Dombrowski, J. Wullbrandt, and P. Krenkel, "Industrie 4.0 in production ramp-up management," Procedia Manuf., vol. 17, pp. 1015-1022, 2018, https://perma.cc/M6GS- 
WFZH.

6. R. Schmitt et al., "On the future of ramp-up management," CIRP J. Manuf. Sci. Technol., vol. 23, pp. 217-225, 2018, https://perma.cc/BR8K-7Y3B.

7. M. Slamanig and H. Winkler, "An exploration of ramp-up strategies in the area of mass customisation,” Int. J. Mass Cust., vol. 4, no. 1/2, pp. 22-43, 2011, https://perma.cc/6HA5N62G.

8. I. Christensen, "New Product Fumbles - Organizing for the Ramp-Up Process," Copenhagen Business School, 2018, https://perma.cc/2C44-ZH6D

9. C. Brecher, S. Storms, C. Ecker, and M. Obdenbusch, "An Approach to Reduce Commissioning and Ramp-up time for Multi-variant Production in Automated Production Facilities," Procedia CIRP, vol. 51, pp. 128-133, 2016, https://perma.cc/GE9L-BTQ6.

10. R. Srinivasan, V. Giannikas, D. McFarlane, and A. Thorne, "Customising with 3D printing: The role of intelligent control," Comput. Ind., vol. 103, pp. 38-46, 2018, https://web.archive.org/web/20201130184417/https://www.sciencedirect.com/science/articl e/abs/pii/S016636151730622X.

11. K. Medini, A., Pierné, J-A., Erkoyuncu, and C., Cornet, “A Model for Cost-Benefit Analysis of Production Ramp-up Strategies," In: Proceedings of APMS 2020 conference, Novi Sad, Serbia, 2020, https://perma.cc/K3B7-PSAW.

12. G. Schuh, W. Stölzle, and F. Straube, "Grundlagen des Anlaufmanagements: Entwicklungen und Trends, Definitionen und Begriffe, Integriertes Anlaufmanagementmodell," In: Anlaufmanagement in der Automobilindustrie erfolgreich umsetzen, pp. 1-6, 2008, https://perma.cc/8QG2-W65G.

13. M. Berg, and K. Säfsten, "Managing Production Ramp-up - Requirement on strategy content," In: Proceedings of POMS 2006 International Conference, Shanghai, China.

14. K. Haapala, K. Kim, G. Kremer, R. Kubat, R. Shilkrot, and F. Sciammarella, "An Open Online Product Marketplace to Overcome Supply and Demand Chain Inefficiencies in Times of Crisis," Smart and Sustainable Manufacturing Systems, vol. 4, no. 3, pp. 299-302, 2020, https://perma.cc/7X67-U7HE.

15. M. Vukovic and K. Weldemariam, "Toward Agile and Resilient Manufacturing Using AI," Smart and Sustainable Manufacturing Systems, vol. 4, no. 3, pp. 330-332, 2020, https://perma.cc/NJ9T-B7Q6.

16. A. Alban, S. E. Chick, D. A. Dongelmans, A. P. J. Vlaar, D. Sent, and S. Group, "ICU capacity management during the COVID-19 pandemic using a process simulation," Intensive Care Med., vol. 46, pp. 1624-1626, 2020, https://perma.cc/R4W6-H3PP.

17. G. Schuh, T. Gartzen, and J. Wagner, "Complexity-oriented ramp-up of assembly systems," CIRP J. Manuf. Sci. Technol., vol. 10, pp. 1-15, 2015, https://perma.cc/DGG8-ZVN7.

18. C. H. Glock and E. H. Grosse, "Decision support models for production ramp-up : a systematic literature review," vol. 53, no. 21, pp. 6637-6651, 2015, https://perma.cc/Q2KXKZYL.

19. K. Szabó, "Ramp-up process improvement practices for time-to-market reduction," Period. Polytech. Soc. Manag. Sci., vol. 26, no. 1, pp. 19-29, 2018, https://perma.cc/NW54-B7GK 
20. C.H. Glock, M.Y. Jaber, and S. Zolfaghari, "Production planning for a ramp-up process with learning in production and growth in demand," International Journal of Production Research, vol. 5, no. 20, pp. 5707-5718, 2012, https://perma.cc/6HY3-E3QM.

21. K.R.N. Hansen, and M. Grunow, "Modelling ramp-up curves to reflect learning: improving capacity planning in secondary pharmaceutical production," International Journal of Production Research, vol. 53, no. 18, pp. 5399-5417, 2015, https://perma.cc/3LUC-VCSV.

22. S. Fjällström, K. Säfsten, U. Harlin, J. Stahre, "Information enabling production ramp-up," Journal of Manufacturing Technology Management, vol. 20, no. 2, pp. 178-196, 2009, https://perma.cc/ABR6-BEM2.

23. A. Drogoul, J. Ferber, C. Cambier, "Multi-agent simulation as a tool for analysing emergent processes in societies," In: Proceedings of Symposium on Approaches to Simulating Social Phenomena and Social Processes, Guilford, UK, 1992.

24. J. Loomis, C. Bond and D. Harpman, "The Potential of Agent-Based Modelling for Performing Economic Analysis of Adaptive Natural Resource Management," Journal of Natural Resources Policy Research, vol. 1, no. 1, pp. 35-48, 2008, https://perma.cc/WV2Q9NYK.

25. M. Herrera, M., Pérez-Hernández, A. Kumar Parlikad, and J. Izquierdo, "Multi-Agent Systems and Complex Networks: Review and Applications in Systems Engineering," Processes, vol. 8, p. 312. 2020, https://perma.cc/U2HS-ARAY.

26. L. Monostori, J. Váncza, and S. R. T. Kumara, "Agent-based systems for manufacturing," CIRP Ann. - Manuf. Technol., vol. 55, no. 2, pp. 697-720, 2006, https://perma.cc/6NCL6GTA.

27. M. G. Avci and H. Selim, "A multi-agent system model for supply chains with lateral preventive transshipments: Application in a multi-national automotive supply chain," Comput. Ind., vol. 82, pp. 28-39, 2016, https://perma.cc/N4WA-LUU9.

28. N. Jennings, K. Sycara, and M. Wooldridge, "A Roadmap of Agent Research and Development," Auton. Agents Multi Agent Syst., vol. 1, no. 1, pp. 7-38, 1998.

29. S. Chehbi, R. Derrouiche, Y. Ouzrout, and A. Bouras, "Multi-Agent Supply Chain Architecture to Optimize Distributed Decision Making," no. July, pp. 1-6, 2003.

30. K. Medini and B. Rabénasolo, "Analysis of the performance of supply chains configurations using multi-agent systems," Int. J. Logist. Res. Appl., vol. 17, no. 6, pp. 441-458, 2014, https://web.archive.org/web/20201130180741/https://www.tandfonline.com/doi/abs/10.108 0/13675567.2014.894183.

31. P. Leitão, "Agent-based distributed manufacturing control: A state-of-the-art survey," Eng. Appl. Artif. Intell., vol. 22, no. 7, pp. 979-991, 2009, https://web.archive.org/web/20201130180938/https://www.sciencedirect.com/science/articl e/abs/pii/S0952197608001437.

32. C. Indriago, O. Cardin, N. Rakoto, P. Castagna, and E. Chacòn, "H2CM: A holonic architecture for flexible hybrid control systems," Comput. Ind., vol. 77, pp. 15-28, 2016, https://web.archive.org/web/20201130181106/https://www.sciencedirect.com/science/articl e/abs/pii/S0166361515300695. 
33. H. Zhang, D. Tang, T. Huang, and C. Xu, "An Agent Based Intelligent Distributed Control Paradigm for Manufacturing Systems," IFAC-PapersOnLine, vol. 49, no. 12, pp. 1549-1554, 2016 ,

https://web.archive.org/web/20201130182630/https://www.sciencedirect.com/science/articl e/pii/S2405896316310825.

34. N. Rodrigues, E. Oliveira, and P. Leitão, "Decentralized and on-the-fly agent-based service reconfiguration in manufacturing systems," Comput. Ind., vol. 101, no. May, pp. 81-90, 2018 ,

https://web.archive.org/web/20201130182753/https://www.sciencedirect.com/science/articl e/abs/pii/S0166361517306991.

35. R. Cupek, A. Ziebinski, L. Huczala, and H. Erdogan, "Agent-based manufacturing execution systems for short-series production scheduling," Comput. Ind., vol. 82, pp. 245-258, 2016, https://web.archive.org/web/20201130182939/https://www.sciencedirect.com/science/articl e/pii/S0166361516301233.

36. S. Doltsinis, P. Ferreira, M. M. Mabkhot, and N. Lohse, "A Decision Support System for rapid ramp-up of industry 4.0 enabled production systems," Comput. Ind., vol. 116, p. 103190,

2020 , https://web.archive.org/web/20201130183022/https://www.sciencedirect.com/science/articl e/abs/pii/S0166361519306876.

37. P. Vrba, O. Harcuba, M. Klíma, and V. Mařík, "Agent-Based Production Scheduling for Aircraft Manufacturing Ramp-up Pavel," Lect. Notes Comput. Sci. (including Subser. Lect. Notes Artif. Intell. Lect. Notes Bioinformatics), vol. 9266, pp. 145-156, 2015, https://web.archive.org/web/20201130183107/https://link.springer.com/book/10.1007\%2F9 78-3-319-22867-9.

38. N. Antzoulatos, E. Castro, D. Scrimieri, and S. Ratchev, "A multi-agent architecture for plug and produce on an industrial assembly platform," Prod. Eng., vol. 8, no. 6, pp. 773-781, 2014 , https://web.archive.org/web/20201130182847/https://link.springer.com/article/10.1007/s11 740-014-0571-x.

39. I. Kovalenko, D. Tilbury, and K. Barton, "The model-based product agent: A control oriented architecture for intelligent products in multi-agent manufacturing systems," Control Eng. Pract., vol. 86, no. March, pp. 105-117, 2019, https://web.archive.org/web/20201130183147/https://www.sciencedirect.com/science/articl e/abs/pii/S0967066118305057.

40. A. Giret, D. Trentesaux, M. A. Salido, E. Garcia, and E. Adam, "A holonic multi-agent methodology to design sustainable intelligent manufacturing control systems," J. Clean. Prod., vol. 167, pp. 1370-1386, 2017, https://web.archive.org/web/20201130183251/https://www.sciencedirect.com/science/articl e/abs/pii/S0959652617305152.

41. C. A. Marín et al., "A conceptual architecture based on intelligent services for manufacturing support systems," Proc. - 2013 IEEE Int. Conf. Syst. Man, Cybern. SMC 2013, no. October, 
pp.

https://web.archive.org/web/20201130183343/https://ieeexplore.ieee.org/document/672256 3.

42. D. T. Meridou, U. Inden, C. P. Rückemann, C. Z. Patrikakis, D. T. I. Kaklamani, and I. S. Venieris, "Ontology-based, multi-agent support of production management," AIP Conf. Proc., $\quad$ vol. 1738, 2016, https://web.archive.org/web/20201130183433/https://aip.scitation.org/doi/abs/10.1063/1.49 $\underline{51834}$.

43. A. Giret, E. Garcia, and V. Botti, "An engineering framework for Service-Oriented Intelligent Manufacturing Systems," Comput. Ind., vol. 81, pp. 116-127, 2016, https://web.archive.org/web/20201130183515/https://www.sciencedirect.com/science/articl e/abs/pii/S0166361516300045.

44. B. Nau, A. Roderburg, F. Klocke, and H. S. Park, "Risk assessment of hybrid manufacturing technologies for ramp-up projects," CIRP J. Manuf. Sci. Technol., vol. 5, no. 4, pp. 228-234, 2012, https://perma.cc/H9XZ-YD75.

45. M. Wooldridge, N. R. Jennings, and D. Kinny, "The Gaia Methodology for Agent-Oriented Analysis and Design," Auton. Agent. Multi. Agent. Syst., vol. 3, no. 3, pp. 285-312, 2000, https://web.archive.org/web/20201130183710/https://link.springer.com/article/10.1023/A:1 010071910869.

46. OMG, "Unified Modeling Language1 (OMG UML1) version 2.5.1," 2017, https://web.archive.org/web/20201130183806/https://www.omg.org/spec/UML/About$\underline{U M L /}$.

47. D.C. Silva, R.A.M. Braga, L.P. Reis, and E. Oliveira, "Designing a meta-model for a generic robotic agent system using Gaia methodology," Information Sciences, vol. 195, pp. 190-210, 2012, https://perma.cc/4F73-SARN.

48. R. Govindu, and R.B. Chinnam, "MASCF: A generic process-centered methodological framework for analysis and design of multi-agent supply chain systems," Computers \& Industrial Engineering, vol. 53, pp. 584-609, 2007, https://perma.cc/BV8M-9TPE.

49. C. Duran-Faundez, M. Ramos, and P. Rodriguez, "Applying Gaia and AUML for the development of multiagent-based control software for flexible manufacturing systems: addressing methodological and implementation issues," Software: Practice and Experience, vol. 45, 12, pp. 1719-1737, 2015, https://perma.cc/UD6T-W8PW.

50. G. Reiner and M. Trcka, "Customized supply chain design: Problems and alternatives for a production company in the food industry. A simulation based analysis," Int. J. Prod. Econ., vol. $89, \quad$ no. $2, \quad$ pp. 2004 , https://web.archive.org/web/20201130183904/https://www.sciencedirect.com/science/articl e/abs/pii/S0925527303000549.

51. F. Bellifemine, A. Poggi, and G. Rimassa, "Developing multi-agent systems with a FIPAcompliant agent framework," Softw. - Pract. Exp., vol. 31, no. 2, pp. 103-128, 2001, https://web.archive.org/web/20201130184017/https://onlinelibrary.wiley.com/doi/abs/10.10 02/1097-024X(200102)31:2\%3C103::AID-SPE358\%3E3.0.CO;2-O. 
52. K. Y. Chen and C. J. Chen, "Applying multi-agent technique in multi-section flexible manufacturing system," Expert Syst. Appl., vol. 37, no. 11, pp. 7310-7318, 2010, https://web.archive.org/web/20201130184112/https://www.sciencedirect.com/science/articl e/abs/pii/S0957417410002952.

53. C. Terwiesch and R. E. Bohn, "Learning and process improvement during production rampup," Int. J. Prod. Econ., vol. 70, no. 1, pp. 1-19, 2001, https://web.archive.org/web/20201130184325/https://www.sciencedirect.com/science/articl e/abs/pii/S0925527300000451. 